\title{
Detection of Driver's Surprised State Based on Blood Pressure and Consideration about Sensitivity of Surprised State
}

\author{
Masayasu Tanaka ${ }^{a}$, Fumiaki Obayashi $^{\mathrm{a}}$, Toshiya Arakawa ${ }^{\mathrm{a}}{ }^{*}$, Shinji Kondo ${ }^{\mathrm{b}}$, Kazuhiro Kozuka ${ }^{\mathrm{a}}$ \\ ${ }^{a}$ Aichi University of Technology, Gamagori, Aichi 443-0047, Japan \\ ${ }^{\mathrm{b}}$ KANDS Inc., Kariya, Aichi 448-0001, Japan \\ *Corresponding Author: arakawa-toshiya@aut.ac.jp
}

\begin{abstract}
The number of deaths from traffic accidents in Japan has been reduced for this 13 consecutive years. One of the reasons is that the collision prevention safety devices for vehicle are developed and equipped many vehicles and it is needed to detect the state of drivers in order to reduce the number of traffic accident more. We are trying to develop this system based on blood pressure and show the efficacy of estimation driver's state based on blood pressure. As a feasibility study, we attempted to estimate driver's state that transits from calm to surprising, which is typical driver's state, based on blood pressure using driving simulator. As a result of experiment, the systolic blood pressure after bicycle rushed out is higher than that before bicycle rushed out and these two data are statistically significant. This means that the systolic blood pressure get to be high when bicycle rushed out thus the feeling of "traffic near-miss" (surprising) can be detected from blood pressure.
\end{abstract}

Keywords: driver's state, estimation, blood pressure.

\section{Introduction}

In this paper, we propose the method of estimating driver's state on driving based on blood pressure.

The number of deaths from traffic accidents in Japan has been reduced for this 13 consecutive years ${ }^{(1)}$. One of the reasons is that the collision prevention safety devices for vehicle are developed and equipped many vehicles. These devices are categorized as two kinds: one is for detecting the state of circumstances around own vehicle; the other is for detecting driver's state. Especially, it is needed to detect the state of drivers, who are the very person controlling vehicle to prevent the traffic accident caused by driver's state, such as excessive tension, inattention, in order to reduce the number of traffic accident more and realize the number of fatal accidents zero.

On considering the detection system of driver's state, we can pick up two kinds systems. One is the camera which monitor the driver's face and expression and warn as sound or light if drivers are not adequate state for driving, like sleepy, tired and so on (Fig. 1(a)) ${ }^{(2)}$. The other is the sensor which monitor the driver's pulse wave and R-R interval and warn as sound or light if drivers are not adequate state for driving, like sleepy, nervous and so on (Fig. 1(b)) ${ }^{(3)}$.

These systems seem to be suitable systems to estimate driver's state, however these have major demerits. Camera system can detect many driver's expression such as angry, depression, sleepy and so on ${ }^{(4)}$, however, this system is high-cost. In addition that, high-spec CPU is needed to execute the algorithm ${ }^{(5)}$ thus this system is inadequate to execute by in-vehicle microcomputer from the view point of spec of microcomputer. The sensor systems that monitor the driver's pulse wave are low-cost and easy to equip to vehicle. Calculation of $\mathrm{R}-\mathrm{R}$ interval is very easy, so high-spec CPU is not needed. The state that the system can detect is limited to a few states, however. In addition that, the pulse wave tends to affected by body motion and it is very difficult to design and develop the filter to reduce the

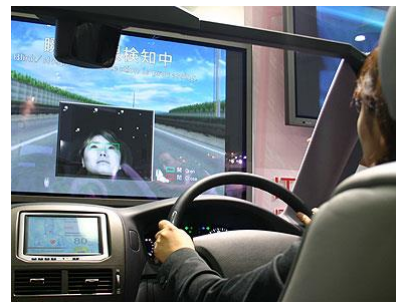

(a)

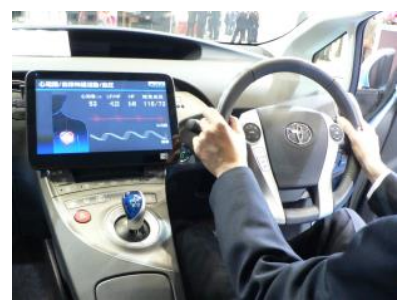

(b)
Fig. 1 Driver state estimation system. affect by body motion. 


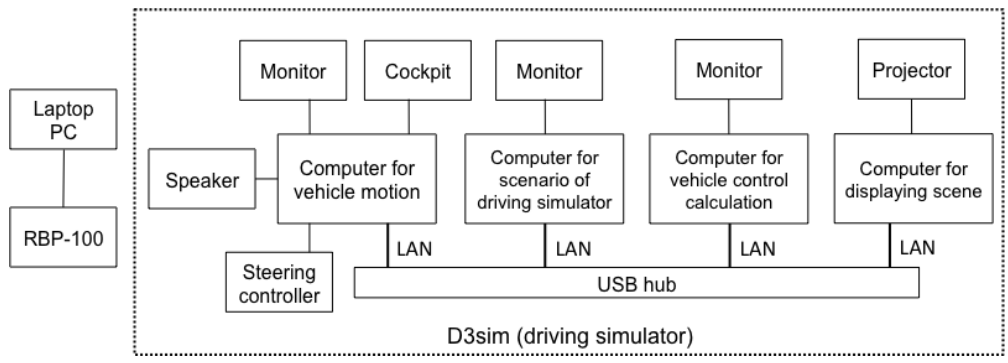

Fig. 2 Experimental system.

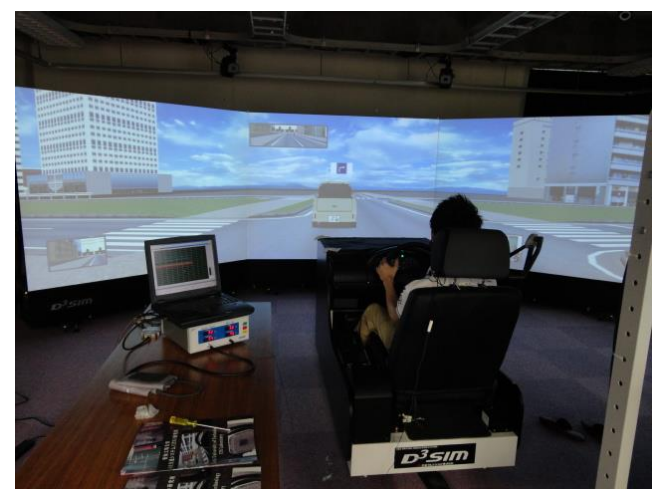

Fig. 3 Experiment image.

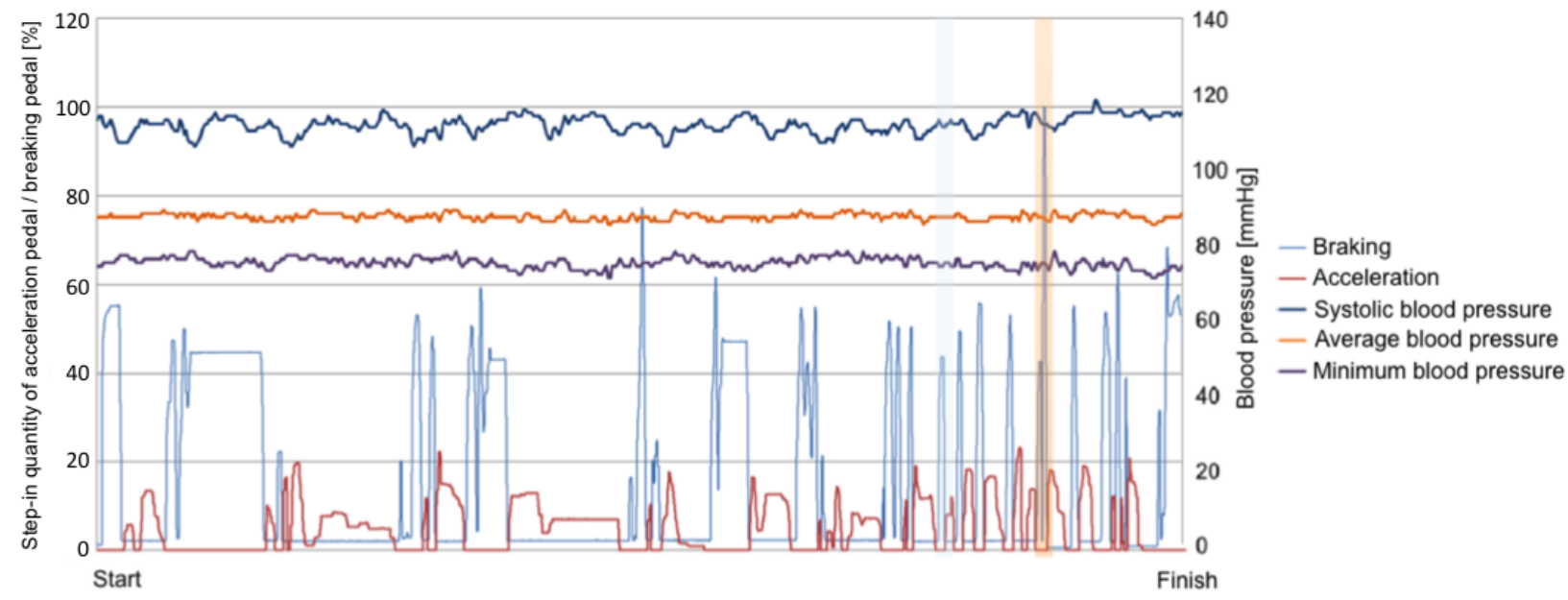

Fig. 4 Time-series data of vehicle control and blood pressure of subject (participant A).

Thus, it is needed to develop another detection system and we conceive the idea of system that can detect driver's state based on blood pressure. The driver's state that can be estimated by blood pressure is a few, however, the number of state that can be estimated by blood pressure system may be larger than that by pulse wave system. In addition that, the effect by body move is less than that of pulse wave system. From above discussion, blood pressure system seems to be adequate in-vehicle system for estimating driver's state.

We are trying to develop this system based on blood pressure and show the efficacy of estimation driver's state based on blood pressure. As a feasibility study, we attempted to detect driver's state that transits from calm to surprising, which is typical driver's state, based on blood pressure.

The paper is consisted as following: Chapter 2 shows the method of experimental and measurement the driver's state. Chapter 3 shows the result of experiment. Chapter 4 shows the consideration based on the result of experiment. Chapter 5 is conclusion and future prospect.

\section{Experiment}

Three male (all males are 21 years old) were joined as a subject of experiment. In order to acquire the data of blood pressure of subject on driving, subject was indicated to drive using driving simulator (D3sim, by Mitsubishi Precision co, LTD.) and measured blood pressure by measurement system (Radia Press RBP-100, by KANDS inc.) on driving. This driving simulator can acquire the data such as velocity, steering angle and acceleration so that we can measure driver's driving behavior. The driving scene is projected to 3 front screens, thus subject can drive as if he drives in real vehicle. 

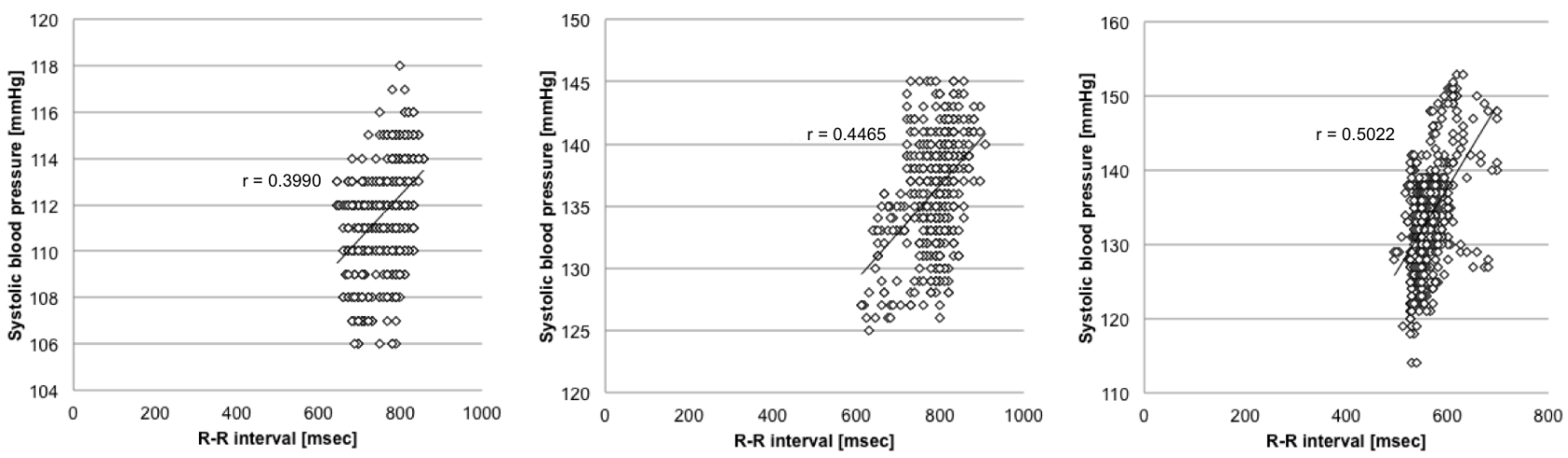

Fig. 5 Correlation between R-R interval and systolic blood pressure. (left: participant A, center: participant B, right: participant C)

All of these participant were not used to drive using driving simulator, thus they drove on a simple course about 8 minutes and were accustomed to driving before experiment (training). After that, they were indicated to drive on a test course using driving simulator (experiment). They drove on a test course about 8 minutes. Their blood pressure and driving behavior, such as velocity, steer angle and acceleration, were measured on driving. A child suddenly rushed out about 5 minutes after start driving, and a bicycle suddenly rushed out about 6 minutes after starting driving, participants may be surprised and change the state from calm to be surprised. Each participants are reported introspective while driving after experiment is finished. The time of a child and bicycle is determined provisionally.

Fig. 2 shows experimental system and Fig. 3 shows the experiment image.

\section{Results}

Fig. 4 shows the subject's blood pressure and driving behavior. In Fig. 4, blue color bar shows the moment that a child rushed out, and orange color bar shows the moment that a bicycle rushed out. Participants stopped a brake pedal strongly at that moment, thus the braking force gets to be strong. Judged from step-in quantity of acceleration pedal and brake pedal, subject drove normally, and irregular behavior was not observed while driving. However, the following characteristics are observed:

(1) Participant A did not notice when a child rushed out, thus he did not step brake pedal then and did not feel surprised.

(2) Participant $\mathrm{C}$ was very surprised when a child rushed out and cannot drive after that calmly for a while.

In addition that, participant $\mathrm{B}$ and $\mathrm{C}$ have some high blood pressure.

\section{Consideration}

\subsection{Detection of driver's surprised state based on the systolic blood pressure}

Firstly, we examined suitability of estimating based on brood pressure so that we examined the correlation R-R interval and systolic blood pressure. It is said that R-R interval detects the nervous or surprised state ${ }^{(6-8)}$, so if $R-R$ interval and systolic blood pressure are correlative, we can say that it is suitable to estimate the surprised state of drivers based on systolic blood pressure. Fig. 5 shows the correlation between R-R interval and systolic blood pressure. From Fig. 5, we can say R-R interval and systolic blood pressure reveal a correlation and it is adequate to estimate surprising state of drivers based on blood pressure, especially systolic blood pressure.

Next, we examined the change of systolic blood pressure before and after a child rushed out. The result is shown in Table 1. In the following, we use "change of systolic blood pressure," which is defined as following:

(change of systolic blood pressure $)=$

(systolic blood pressure) -

(systolic blood pressure on starting experiment)

The reason that we defined "Relative systolic blood pressure" is that the blood pressure is different each participants so that absolute value of systolic blood pressure is useless and the relative value of systolic blood pressure is important.

From Table 1, relative systolic blood pressure of all

Table 1 Relative systolic blood pressure before and after a child rushed out. 


\begin{tabular}{ccc}
\hline \hline & $\begin{array}{c}\text { Before a child } \\
\text { rushed out }\end{array}$ & $\begin{array}{c}\text { After a child } \\
\text { rushed out }\end{array}$ \\
\hline Participant A & -1.84 & -1.05 \\
Participant B & 6.68 & 10.05 \\
Participant C & 5.58 & 17.54 \\
\hline \hline
\end{tabular}

pressure after a child rushed out gets to be higher than that before a child rushed out.

However, when drivers get to be surprised really? In order to consider this, Fig. 6 shows the average and standard deviation of relative systolic blood pressure of each 5 minutes from 10 seconds before a child rushed out to

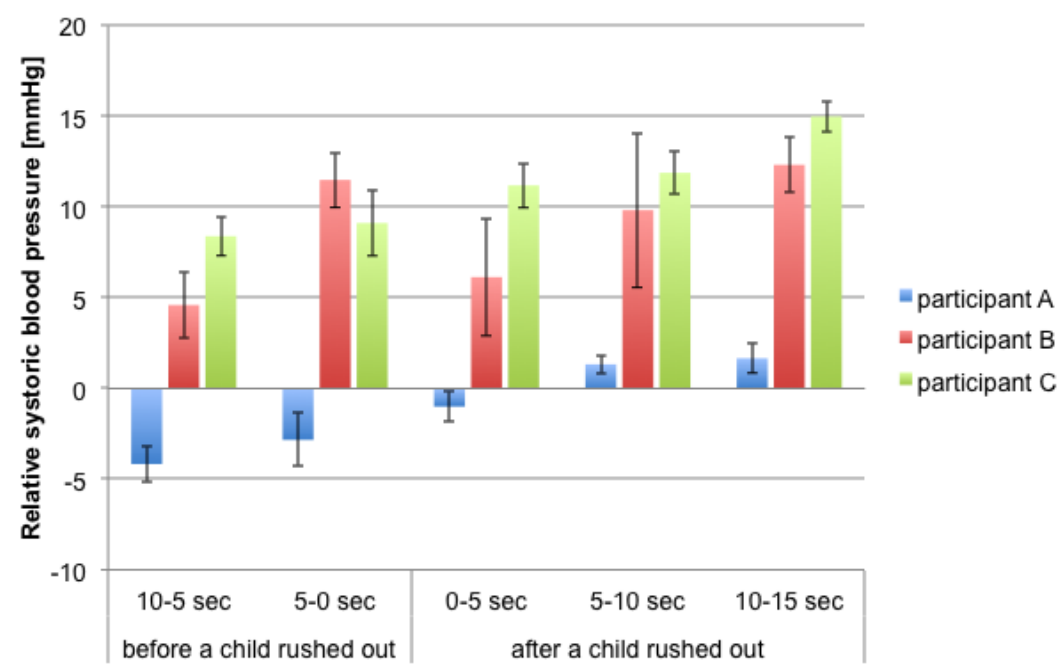

Fig. 6 Relative systolic blood pressure of each 5 seconds from 10 seconds before a child rushed out and 15 seconds after a child rushed out.

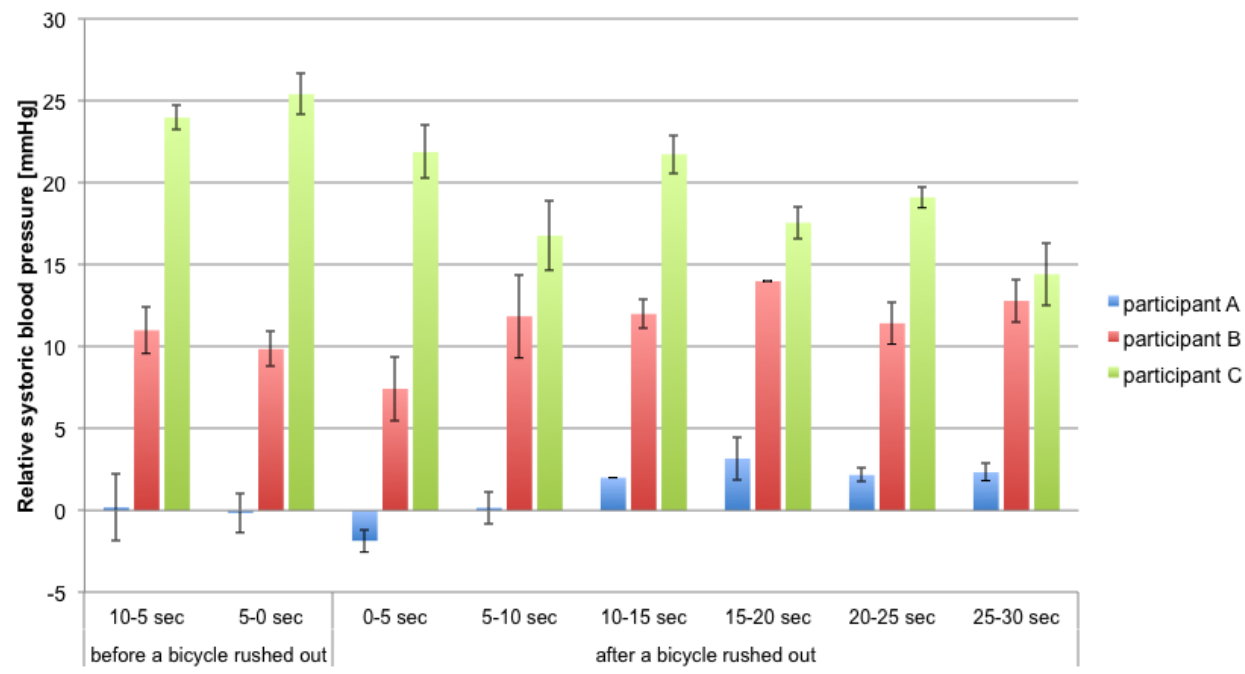

Fig. 7 Relative systolic blood pressure of each 5 seconds from 10 seconds before a bicycle rushed out and 30 seconds after a bicycle rushed out.

participants after a child rushed out gets to be higher than that before a child rushed out. This means that the systolic blood pressure is reflected the surprises state of drivers, in other words, surprised state of drivers can be detected by the systolic blood pressure.

\subsection{About sensitivity of surprised state}

From Table 1, we showed that relative systolic blood
20 seconds after a child rushed out. From Fig. 6, relative systolic blood pressure does not get to be high immediately after a child rushed out and get to be high gradually. Thus, drivers feel surprised from the point of blood pressure not immediately after a child rushed out but a little later, specifically 15 seconds after from Fig. 6.

Next, Fig. 7 shows the average and standard deviation of relative systolic blood pressure of each 5 minutes from 
10 seconds before a bicycle rushed out to 30 seconds after a bicycle rushed out. From Fig. 7, as with Fig. 6, relative systolic blood pressure does not get to be high immediately after a bicycle rushed out and get to be high gradually. However, relative systolic blood pressure before a bicycle rushed out is higher than that after a bicycle rushed out in the case of participant $\mathrm{C}$ because participant $\mathrm{C}$ is crushed wall after a child rushed out so that he was very surprised and his relative systolic blood pressure was relatively high before a bicycle rushed out.

It is found that the relative systolic blood pressure is highest between 10 seconds and 20 seconds after a bicycle rushed out when relative systolic blood pressure of all participants are compared. Thus, drivers feel surprised from the point of blood pressure not immediately after a bicycle rushed out but a little later, between 10 seconds and 20 seconds after from Fig. 7.

\section{Conclusions}

We detected the driver's state, especially surprised state, based on blood pressure and analyzed the change of relative blood pressure. It is found that it seems to be able to detect the driver's state based on blood pressure and drivers feel surprised from the point of blood pressure not immediately after near-miss event but a little later of near-miss event. From this result, for example, it may be effective to calm drivers down by HMI or driving support a little later that surprised state is detected.

In this paper, as a feasibility study, we tried to detect the driver's surprised state based on blood pressure. However, it may be able to detect other driver's state besides surprised state based on blood pressure. Considering results of this experiment, we will develop driver's state estimating system based on blood pressure.

\section{References}

(1) Cabinet Office of Japan, White Paper On Traffic Safety In Japan 2014 edition (2014).

(2) ITmedia Lifestyle, "The system which gauge drivers' feeling", http://www.itmedia.co.jp/lifestyle/articles/0510/20/new s085.html (2013).

(3) NIKKEI Digital Health, The system that measures electrocardiogram and a pulse wave with a steering, and predicts the condition sudden change on driving, http://www.nikkeibp.co.jp/article/dho/20130326/34522
8/ (2013).

(4) Ministry of Land, Infrastructure, Transport and Tourism (MLIT), the present situation of safety measures of motor coach (2014).

(5) J. Iio, A. Date: MACAO \& FacePoint: The Face Detection System, Proceedings of linux conference 2003 (2003).

(6) Keita Takahashi et al.: A Quantitative Analysis on Stress and Fatigue for VDT Workers Measured by Heart Rate Variability (HRV), Scientific and Technical Reports of Graduate School of Engineering and Resource Science, Akita University, No. 34, pp. 1-7 (2009).

(7) Masatoshi Yanagidaira, Mitsuo Yasushi: Development of Driver's Condition Monitor, Pioneer R\&D, Vol. 13, No. 2 (2013)

(8) Masatoshi Yanagidaira, Mitsuo Yasushi: Development of Driver's Condition Monitor, Pioneer R\&D, Vol. 14, No. 3 (2013) 